\title{
Descrição do Comportamento de Superfície do Boto Cinza, Sotalia guianensis, na Praia de Pipa - RN
}

\author{
Description of the Surface Behaviour of Marine Tucuxi, Sotalia guianensis, \\ at Pipa Beach - RN
}

\author{
Lídio França do Nascimento*, Priscila Izabel A. P. Medeiros \& Maria Emilia Yamamoto \\ Universidade Federal do Rio Grande do Norte, Natal, Brasil
}

\begin{abstract}
Resumo
O estudo do comportamento animal é uma área de pesquisa consolidada, que cresceu e se diversificou em vários países e que deu origem a disciplinas e abordagens de investigação, como a Etologia, a Ecologia Comportamental, a Neuroecologia, a Psicologia e a Psicologia evolucionista. No estudo do comportamento animal, o etograma é a base para estudos comportamentais de espécies pouco conhecidas, como também para comparar comportamentos de populações distintas de uma mesma espécie. Tradicionalmente, um etograma descreve de forma detalhada eventos comportamentais realizados por indivíduos de uma espécie. O presente estudo apresenta um etograma dos eventos comportamentais realizados na superfície por uma espécie de golfinho costeiro, boto cinza, na praia de Pipa - RN.

Palavras-chave: Etograma; Sotalia guianensis; comportamento de superfície.
\end{abstract}

\begin{abstract}
Research in animal behavior is a consolidated area whose development and diversification in several countries have originated some subjects and investigation approaches, such as Ethology, Behavior Ecology, Neuroecology, Psychology and Evolutionist Psychology. In the study of animal behavior, the ethogram is the foundation for studies on behavior patterns of distinct populations of the same species. An ethogram traditionally describes behavioral events performed by individuals of the same species in detail. This study presents an ethogram of behavioral events performed on surface by a costal dolphin known as the gray dolphin (Marine Tucuxi Dolphin), at Pipa Beach, RN.

Keywords: Ethogram; Sotalia guianensis; surface behaviour.
\end{abstract}

O estudo comportamento animal é uma área de pesquisa e investigação consolidada em países europeus e nos Estados Unidos, fundada por Konrad Lorenz e Nikolaas Tinbergen, por volta de 1930 na Europa (Alcock, 2003; Yamamoto \& Volpato, 2007). Segundo Shettleworth (2001), o estudo do comportamento animal surgiu com o objetivo de estudar comportamentos inatos de espécies em seus respectivos ambientes naturais, ao contrário da perspectiva comportamentalista (behaviorismo), que realizava estudos com o comportamento animal em laboratório com algumas poucas espécies (Bateson, 2003). Desde então, o estudo do comportamento animal cresceu e se diversificou, dando origem a várias disciplinas e abordagens de investigação, como a própria Etologia, a Ecologia Comportamen-

\footnotetext{
*Endereço para correspondência: Universidade Federal do Rio Grande do Norte, Centro de Biociências, Departamento de Fisiologia, Campus Universitário, Natal, RN, 59078-970. Caixa Postal 1511.E-mail: tkdelphinus@yahoo.com.br.Faculdade de Excelência Educacional do Rio Grande do Norte, Rua Doutor Hernan Hugo Gomes, 90, Capim Macio, Cep 59082-270, Natal, RN. Nós gostaríamos de agradecer aos membros do projeto Pequenos Cetáceos (UFRN), à Coordenação de Aperfeiçoam de pessoal de nível superior (CAPES), à Suelaine V. M. de Moraes, Sathyabama Chellapa e Paulo Simões-Lopes pelas sugestões sobre o manuscrito.
}

tal, a Neuroecologia, a Psicologia e a Psicologia evolucionista (Yamamoto \& Volpato, 2007). No Brasil, o estudo do comportamento animal teve sua origem na psicologia, na década de 60, no Estado de São Paulo, irradiando-se depois pelo país (Yamamoto \& Volpato, 2007).

No estudo do comportamento animal, o etograma geralmente é o primeiro passo que o pesquisador dá no processo de coleta e quantificação de informações sobre o comportamento de uma espécie (Freitas \& Nishida, 2007). Tradicionalmente, um etograma descreve de forma detalhada eventos comportamentais realizados por indivíduos de uma determinada espécie (Muller, Boutiere, Weaver, \& Candelon, 1998). O etograma é a base para estudos comportamentais de espécies pouco conhecidas, como também para comparar padrões comportamentais de populações distintas de uma mesma espécie (Barlow, 1977). Essa ferramenta vem sendo usada no estudo do comportamento de espécies, principalmente daquelas com as quais nós somos mais familiarizados, por exemplo, primatas (Stevenson \& Poole, 1976), aves (Ellis, Kepler, Swengel, \& Archibald, 1998) e lagartos (Torr \& Shine, 1994).

Nos cetáceos (baleias, botos e golfinhos), além da inacessibilidade do ambiente aquático, o próprio comportamento 
desses animais é um obstáculo a ser superado na construção de um etograma. Eles podem se deslocar muitos quilômetros por dia (Norris \& Dohl, 1980) e passar grande parte do tempo submersos, sendo visíveis apenas por pequenos intervalos de tempo (Tayler \& Sayman, 1973). Apesar disso, etogramas de algumas espécies, principalmente aquelas de hábitos costeiros, foram realizados (Martinez \& Klinghammer, 1969; Miles \& Herzing, 2003; Muller et al., 1998; Slooten, 1994).

O boto cinza, Sotalia guianensis, é um golfinho de hábito costeiro, freqüentando baías, enseadas e estuários (Silva \& Best, 1996). Ao longo da distribuição da espécie, algumas populações são consideradas residentes (Flores, 1999; Pizzorno, Siciliano, \& Simão, 1999), freqüentando uma mesma área ao longo do ano. Apesar desses fatores (a condição de residentes e o hábito costeiro) favorecerem o desenvolvimento de estudos comportamentais em outras espécies de cetáceos, no boto cinza, entretanto, estudos descritivos do comportamento de superfície ainda são escassos.

Desta forma, o objetivo do presente trabalho foi elaborar um etograma através da descrição das atividades comportamentais realizadas na superfície pelo boto cinza em seu ambiente natural, fornecendo assim, subsídios para estudos futuros com essa população, assim como, para estudos comparativos com outras populações dessa e de outras espécies de golfinhos.

\section{Método}

O estudo foi desenvolvido na Praia de Pipa em duas enseadas, a enseada do Madeiro (06o 13' 23,6"S; $35^{\circ} 04^{\prime}$ $14,3^{\prime \prime}$ w) e a enseada do Curral (06²'13'36.7" S; 35³’36.7" W), no litoral sul do Estado do Rio Grande do Norte. Os animais foram monitorados de pontos fixos, de 6 a 12 horas diárias, por dois pesquisadores usando binóculos $(10 \mathrm{x}$ $50 \mathrm{~mm}$, Field $7,5^{\circ}$ ) e gravadores portáteis (Aiwa TP-VS 480). Os pesquisadores passaram por um treinamento até que o índice de concordância na identificação dos eventos comportamentais e das faixas etárias fosse de pelo menos 85\%. O estudo foi realizado entre os anos de 1999 a 2004. $\mathrm{Na}$ enseada do curral foram realizados 151 dias (863,9 horas de observações) e na enseada do Madeiro 72 dias (364,1 horas de observações), totalizando 223 dias (1228 horas) de observações ao longo desses anos. Os métodos de registros usados foram o Ad libitum, animal focal e grupo focal (Altmann, 1974). A descrição dos comportamentos foi baseada apenas nos eventos comportamentais realizados na superfície da água ou logo abaixo dela (Slooten, 1994).

\section{Definições Adotadas}

Foram adotados os termos evento comportamental para ações comportamentais simples, de curta duração e categoria comportamental, como sendo um conjunto dessas ações de curta duração (Altmann, 1974). As nomenclaturas usadas para os eventos e categorias comportamentais em alguns casos foram adotadas e/ou adaptadas de estudos com outras espécies de golfinhos (Muller et al., 1998; Slooten, 1994) e em alguns casos, essas nomenclaturas foram criadas.
Por estimativa visual, os animais foram categorizados como adultos, juvenis e filhotes, de acordo com o tamanho e coloração. Consideram-se juvenis animais que medem até $3 / 4$ do cumprimento total do adulto e filhotes medem 1/4 do tamanho do adulto e possuem uma coloração cinzarosada (Geise, Gomes, \& Cerqueira, 1999). O filhote geralmente é acompanhado de um animal adulto (Lodi, 2003). Um grupo foi definido quando dois ou mais animais estavam dentro das enseadas ao mesmo tempo. Este critério foi escolhido porque observações prévias têm mostrado que animais mesmo distantes, podem interagir ou entrar e sair das enseadas juntos.

\section{Resultados}

Um total de 30 eventos comportamentais foi registrado e dividido entre sete categorias comportamentais (Tabela 1): atividade aérea, alimentação, brincadeira social, brincadeira com objetos, comportamento agressivo, comportamento sexual e locomoção. Essas categorias foram descritas da seguinte forma:

Atividade aérea. Exposição total ou parcial do corpo do animal acima da superfície da água, exceto a subida para respiração.

Alimentação. Deslocamentos rápidos, posicionamento ventral próximo à superfície da água; peixes podem ser vistos saltando em fuga.

Brincadeira social. Interações com ou sem contato físico entre dois ou mais animais com repetição exagerada, em intensidade ou freqüência de um evento comportamental.

Brincadeira com objetos. Interações com objetos através de arremessos com a boca e nadadeira caudal e carregar o objeto com a nadadeira peitoral e/ou dorsal.

Comportamentos agonísticos. Mordidas e golpes com a nadadeira caudal ou com a cabeça desferidos em conespecifícos.

Comportamento sexual. Contato ventro-ventral de dois animais adultos.

Natação. Movimento constante em uma determinada direção, de uma área para outra, com intervalos regulares para respiração.

$\mathrm{Na}$ atividade aérea foram registrados os seguintes eventos: três tipos de saltos; dois tipos de espiar; quatro tipos de batidas com o corpo. Na alimentação: perseguição, giros de $360^{\circ}$, bote e consumação da presa. Foi registrado um tipo de comportamento social, a potencial cópula; Comportamentos agressivos: mordida, golpe com cauda e golpe com cabeça; Brincadeiras com objetos: jogando objeto com a boca, carregando objeto com a peitoral, carregando objeto com a dorsal, Brincadeira social: surf e impulsão de filhote. Na locomoção: lenta, rápida, deslizando na superfície e natação com saltos.

\section{Discussão}

Segundo Lodi (2003), o boto cinza possui um comportamento tímido, receoso e arredio, e não tem o hábito de nadar na proa de embarcações como outras espécies de golfi- 
Nascimento, L. F., Medeiros, P. I. A. P., \& Yamamoto, M. E. (2008). Descrição do Comportamento de Superfície do Boto Cinza, Sotalia guianensis, na Praia de Pipa - RN.

Tabela 1

Descrição dos Eventos Comportamentais Realizados pelo Boto Cinza na Praia de Pipa

\section{Categoria comportamental / Evento} Atividade aérea

\section{Descrição dos eventos comportamentais}

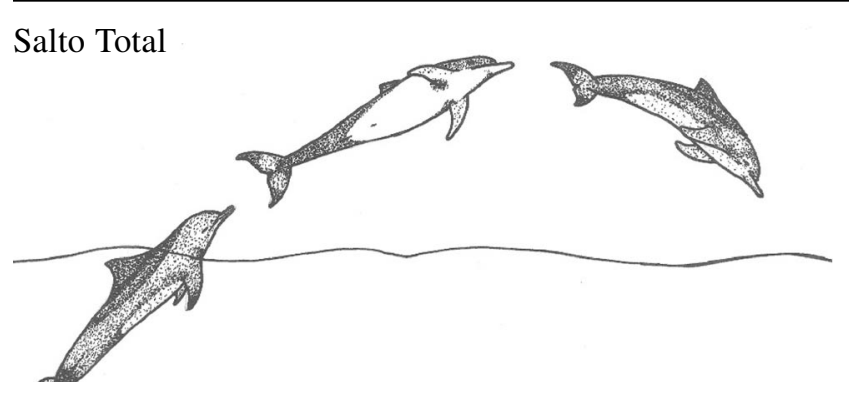

Exposição total do corpo, acima da superfície da água.

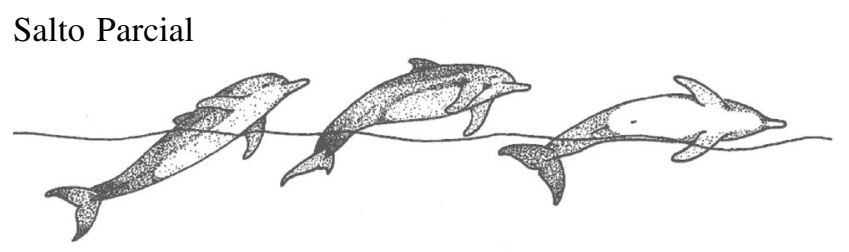

A exposição do corpo não é totalmente observada acima da superfície da água.

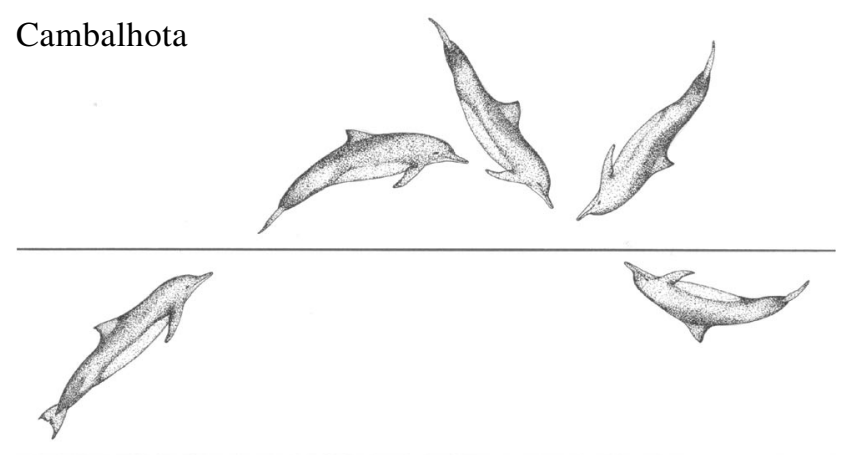

No qual o animal executa um giro no seu próprio eixo, retornando à água com a região ventral ou lateral do corpo.

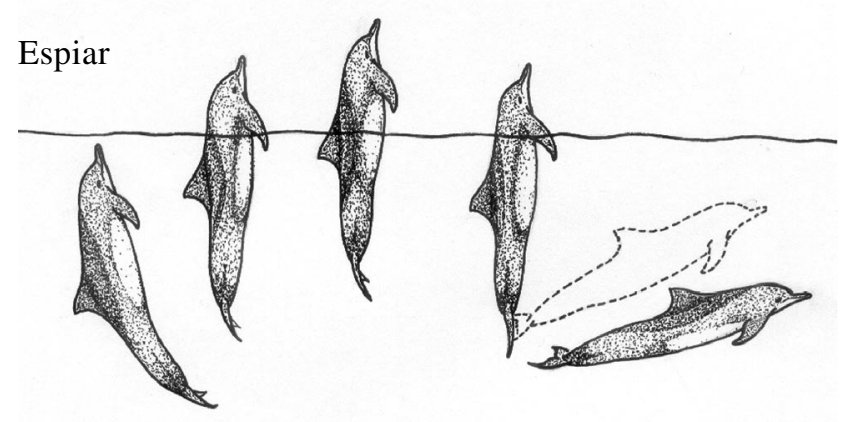

$\mathrm{O}$ animal emerge verticalmente, fazendo um ângulo de $90^{\circ}$ em relação à superfície da água.

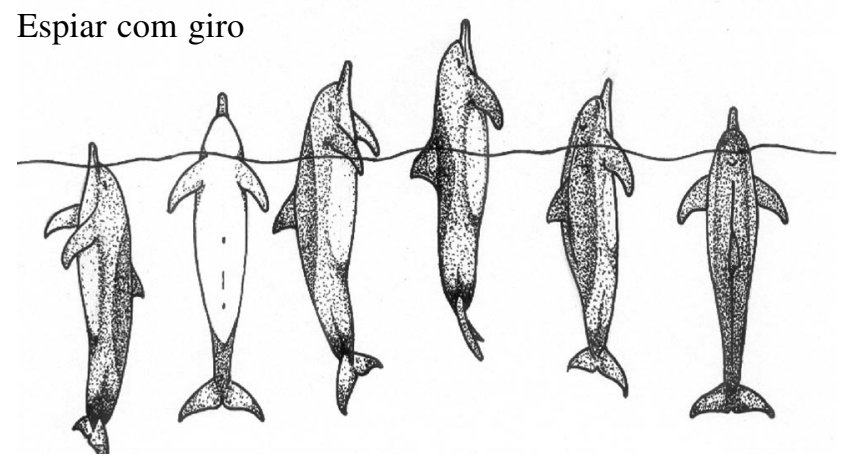

O animal emerge verticalmente, fazendo um ângulo de $90^{\circ}$ com a superfície da água, realizando um giro no seu próprio eixo. 


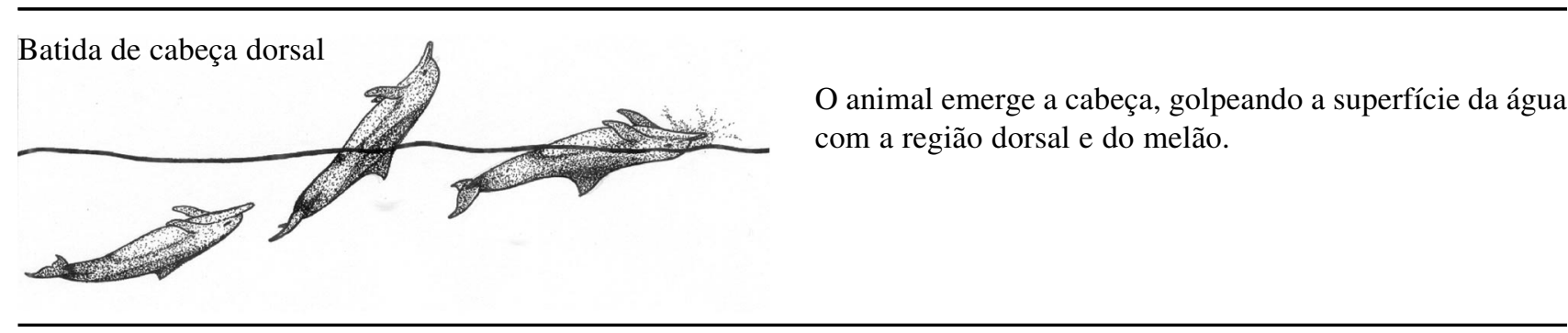

Batida de cabeça inversa

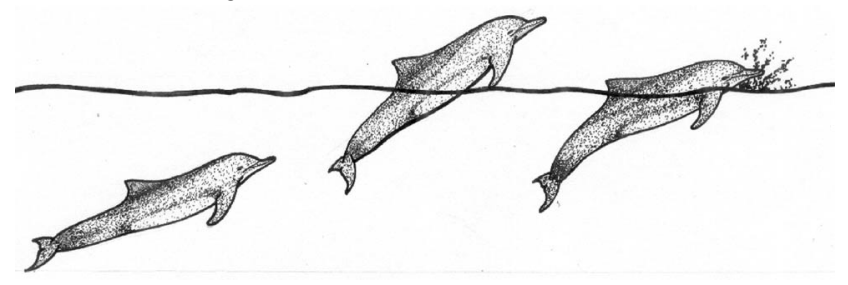

O animal emerge cabeça, golpeando a superfície da água, com a mandíbula.

Batida com a cauda

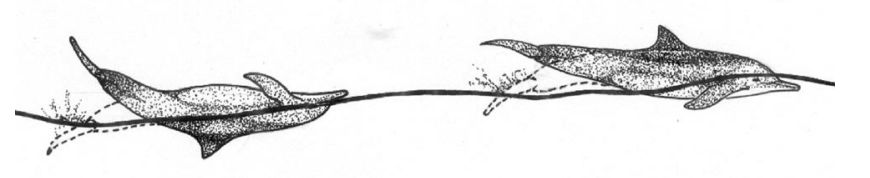

Posicionado dorsal ou ventralmente, o animal ergue o pedúnculo e a nadadeira caudal golpeando a superfície da água.

\section{Forrageio}

\section{Descrições}

\section{Perseguição}

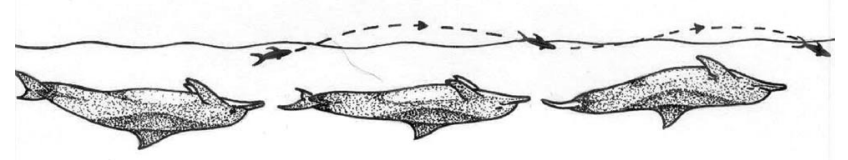

É caracterizada por um deslocamento em velocidade, rente à superfície da água, com o animal nadando com a região ventral para cima.

Giros de $360^{\circ}$

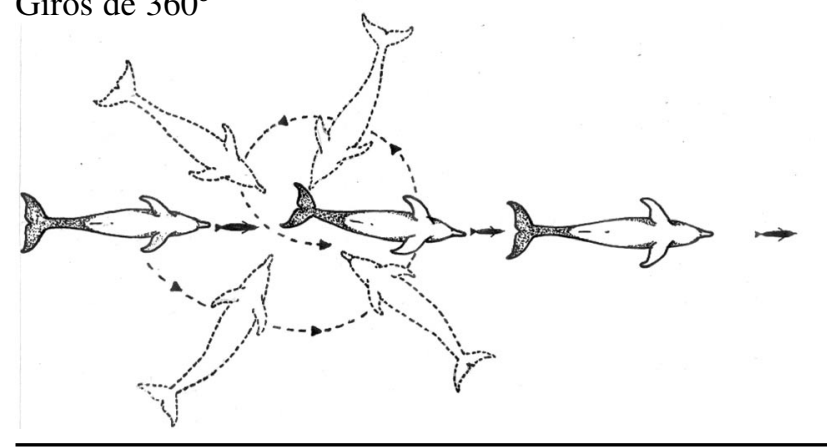

São realizados durante a perseguição, estando o animal posicionado com a região ventral para cima.

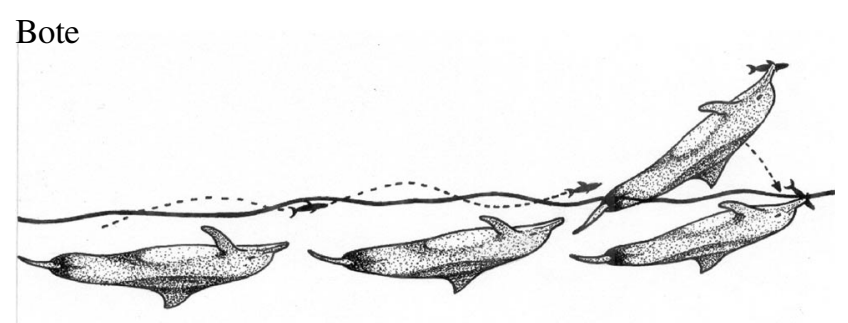

É a finalização da perseguição, no qual o animal consegue capturar a presa em pleno ar, expondo parte do seu corpo. 
Nascimento, L. F., Medeiros, P. I. A. P., \& Yamamoto, M. E. (2008). Descrição do Comportamento de Superfície do Boto Cinza, Sotalia guianensis, na Praia de Pipa - RN.

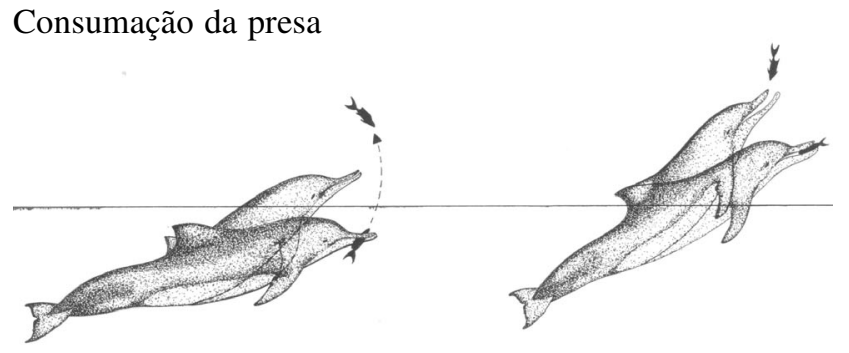

Locomoção
Após a captura, a presa é jogada ao ar, ou na superfície da água, algumas vezes, sendo recapturada e em seguida, engolida.

\section{Rápida}

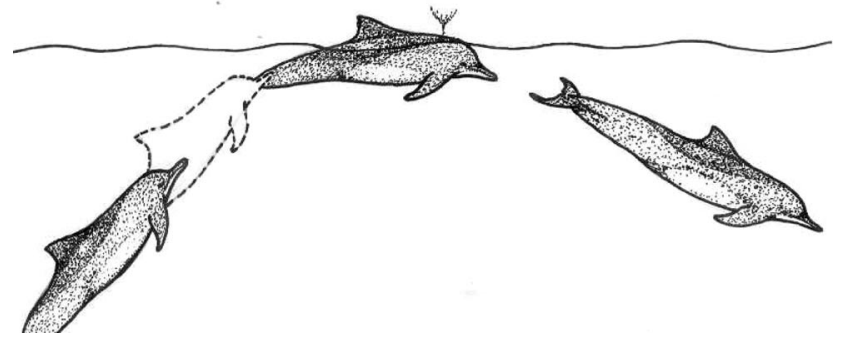

No deslocamento rápido, observa-se a mesma seqüência do deslocamento lento, diferenciando-se apenas pelo movimento mais rápido e com um arqueamento do corpo mais acentuado.

Deslizando na superfície

Este deslocamento se difere dos outros, devido ao animal permanecer por mais tempo na superfície da água em velocidade, criando um rastro de espuma.

\section{Com saltos}

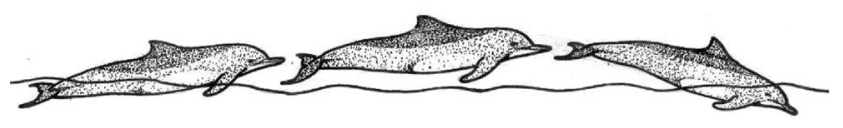

Este comportamento é caracterizado por uma seqüência de saltos, rentes à superfície da água. 


\section{Comportamentos Sexual}

Potencial cópula

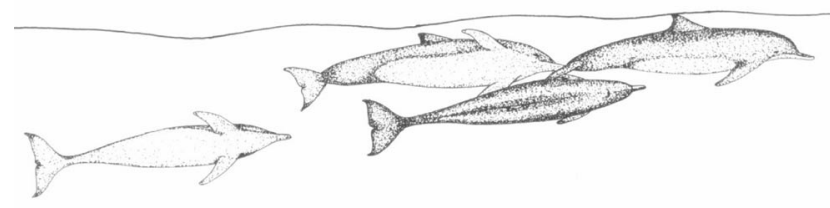

Animais adultos em intenso contato físico com as regiões ventrais.

\section{Comportamentos agonísticos}

\section{Golpe com a cauda}

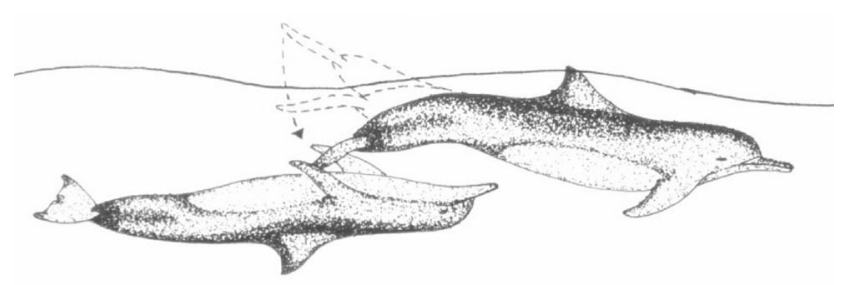

Animal golpeia outro animal com a nadadeira caudal.

Golpe com a cabeça

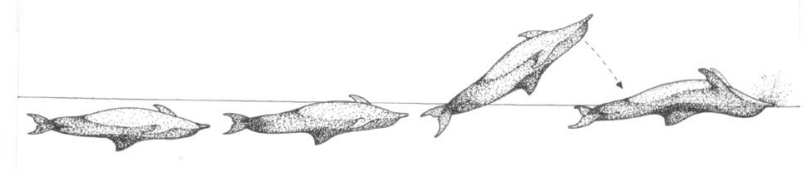

Animal golpeia outro animal com a cabeça.

\section{Mordida}

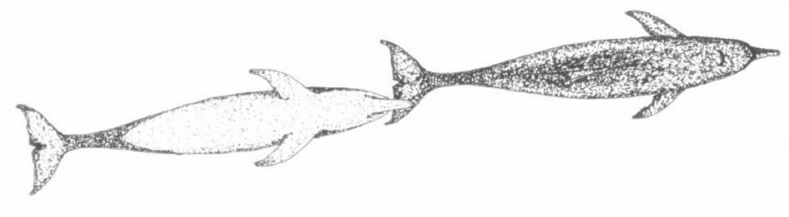

Mordidas desferidas em conespecifícos.

\section{Brincadeiras}

Brincadeiras com objetos

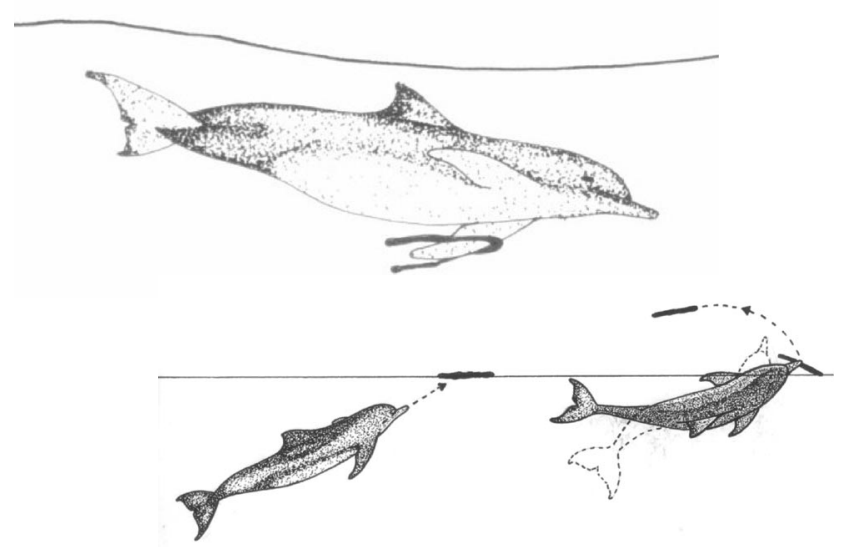

Os animais podem interagir com objetos, através da boca, arremessando-os, carregando em suas nadadeiras peitorais ou dorsais. 
Nascimento, L. F., Medeiros, P. I. A. P., \& Yamamoto, M. E. (2008). Descrição do Comportamento de Superfície do Boto Cinza, Sotalia guianensis, na Praia de Pipa - RN.

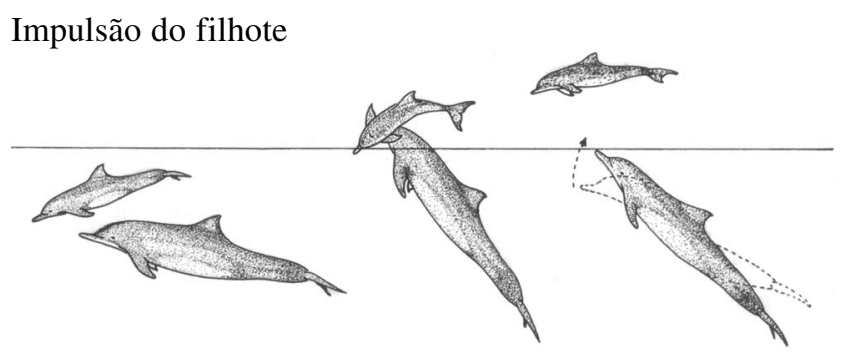

Um animal é impulsionado para fora da água por outro, usando o rosto.

Surf

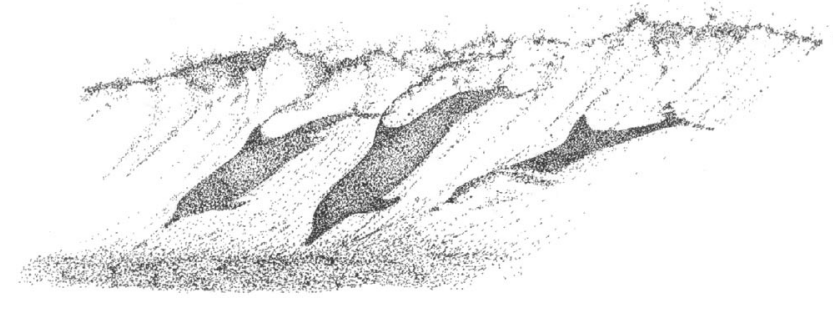

Ocorre quando um animal tira vantagem do movimento das ondas para se mover, saltos podem ser observados.

nhos. Entretanto, em algumas áreas, o boto cinza apresentou um repertório comportamental de superfície tão variado e complexo (Geise et al., 1999; Hayes, 1999; Nascimento, 2006) quanto os realizados por outras espécies de golfinhos conhecidos pela plasticidade comportamental, como o golfinho nariz de garrafa (Muller et al., 1998), e o golfinho rotador (Norris \& Dohl, 1980).

Alguns comportamentos descritos no presente estudo não estão presentes apenas nas populações do boto cinza estudadas, mas também fazem parte do repertório comportamental de outras espécies de botos, golfinhos e baleias, evidenciando que existe uma generalização de alguns comportamentos para a ordem cetácea. Eventos comportamentais como os saltos, espiar (spyhop) e batidas são descritos em algumas áreas nos quais o boto cinza foi estudado (Geise et al., 1999; Hayes, 1999) e em outras espécies de cetáceos (Martinez \& Klinghammer, 1969; Muller et al., 1998; Norris \& Dohl, 1980).

Por outro lado, alguns comportamentos podem ocorrer em poucas áreas e até mesmo serem endêmicos a uma determinada população. Essa hipótese fica mais evidente quando analisamos a descrição do comportamento alimentar ou das estratégias de caça do boto cinza em algumas áreas. Estratégias de alimentação distintas são descritas para o boto cinza ao longo da distribuição da espécie (Domit, 2006; Monteiro, Souto, \& Nascimento, 2006; Nascimento, 2006; Rossi-Santos, 1997). Essas diferenças nas estratégias alimentares entre essas populações podem ser conseqüências das características oceanográficas e fisiográficas de cada área, atividades antrópicas (adaptação à atividade pesqueira) e principalmente pela concentração, distribuição e a ecologia comportamental do recurso alimentar nessas áreas (Wedekin et al., 2003). Assim, as estratégias de alimentação de populações distintas do boto cinza e de outras espécies de cetáceos seriam moldadas pelas adaptações e contra-adaptações comportamentais entre presa e predador em seus respectivos habitat. Segundo SimõesLopes (2005), os recursos alimentares e os fatores ambientais geram necessidades ecológicas particulares, podendo favorecer uma estratégia de sobrevivência em detrimento da outra.

Alguns eventos do repertório comportamental aéreo do boto cinza registrados em Pipa, também foram registrados em outras populações do boto cinza. Eventos como saltos totais, parciais e o espiar são comuns entre as populações da espécie (Geise et al., 1999; Hayes, 1999). Entretanto, em Pipa, foram registrados eventos ainda não descritos para outras populações do boto cinza, como o espiar com giro e a impulsão de filhote, descrita por Spinelli, Nascimento e Yamamoto (2002).

O comportamento sexual é difícil de ser identificado em golfinhos que habitam águas escuras como o boto cinza. Segundo Simões-Lopes (2005), nos cetáceos, a cópula propriamente dita é breve, com eventos rápidos e difíceis de serem detectados na natureza. Entretanto, o autor fala que em algumas espécies tropicais, que vivem em águas transparentes, como o golfinho rotador, a cópula é facilmente detectável. Assim, optamos por usar o termo potencial cópula para designar os comportamentos nos quais botos adultos executavam contatos ventro-ventrais, um indicador para a cópula (Simões-Lopes, 2005), por dois motivos. Primeiro, as águas da praia de Pipa são turvas, impossibilitando a visualização da ereção e retração do pênis, para dentro da fenda genital do macho. Segundo, o boto cinza não apresenta dimorfismo sexual (diferenças morfológicas entre machos e fêmeas). Esse contato ventro-ventral pode estar ocorrendo em outro contexto como sugere Simões-Lopes (2005). Segundo o autor, esse com- 
portamento pode acontecer em disputas hierárquicas entre machos. Ele ainda afirma que no estudo do comportamento animal esse comportamento entre animais do mesmo sexo é chamado de "montada", comportamento comum em mamíferos terrestres, como primatas e canídeos.

De uma forma geral, em espécies terrestres, a exposição de dentes, a ereção dos pelos (pilo ereção) e vocalizações demonstram um comportamento agressivo (exibições de ameaça). Em golfinhos, os comportamentos agressivos não são tão explícitos como em espécies terrestres. Alguns eventos comportamentais aéreos, como batidas de cabeça, batidas com a nadadeira caudal e peitoral na superfície da água produzem um forte som e podem ser direcionados a outros golfinhos como uma ameaça (Simões-Lopes, 2005). Na praia de Pipa, os botos realizaram investidas, golpes com a cabeça, mordidas, batidas de cauda e de cabeça nas interações com contato físico entre animais adultos e na tentativa de aproximação de banhistas a grupos de botos com filhotes, no qual, animais adultos se aproximavam dos banhistas e executavam batidas de caudais seqüenciais na superfície.

Interações com objetos que ocorreram em Pipa são similares a eventos descritos para outras espécies de cetáceos, como o golfinho de Hector (Slooten, 1994), a baleia da Groenlândia (Würsig, Dorsey, Richardson, \& Wells, 1989) e o golfinho pintado do Atlântico (Miles \& Herzing, 2003). Os animais podem interagir com objetos, através da boca, arremessando-os, carregando em suas nadadeiras peitorais e/ou dorsal. Interações com objetos também foram descritos por Spinelli et al. (2002) com o boto cinza na praia de Pipa.

Esperamos que o presente estudo enriqueça o pouco que sabemos sobre o comportamento de superfície do boto cinza em seu ambiente natural, e que este etograma sirva de base para outros estudos dessa população, assim como, para estudos comparativos do comportamento entre populações do boto cinza e até mesmo entre espécies distintas.

\section{Referências}

Alcock, J. (2003). A text book history of animal behaviour. Animal Behaviour, 65, 3-10.

Altmann, J. (1974). Observational study of behaviour: Sampling methods. Behaviour, 49, 227-265.

Barlow, G. W. (1977). Modal action patterns. In T. A. Sebeok (Ed.), How animals communicate (pp. 98-134). Bloomington, IN: Indiana University Press.

Bateson, P. (2003). The promise of behavioural biology. Animal Behaviour, 65, 11-17.

Domit, C. (2006). Comportamento de pesca do Boto-cinza "Sotalia guianensis" (van Bénedén, 1864). Dissertação de Mestrado em Zoologia não-publicada, Universidade Federal do Paraná, Curitiba, PR.

Ellis, D. H., Kepler, C. B., Swengel, S. R., \& Archibald, G. W. (1998). A sociogram for the cranes of the world. Behavioral Processes, 43, 125-151.

Flores, P. A. C. (1999). Preliminary results of a photoidentification study of the marine tucuxi Sotalia fluviatilis in southern Brazil. Marine Mammals Science, 15, 840-847.
Freitas, E. G., \& Nishida, S. M. (2007). Métodos de estudos do comportamento animal. In M. E. Yamamoto \& G. L. Volpato, Comportamento animal: Vol. 1 (pp. 39-64). Natal, RN: Editora da Universidade Federal do Rio Grande do Norte.

Geise, L., Gomes, N., \& Cerqueira, R. (1999). Behaviour, habitat use and population size of Sotalia fluviatilis (Gervais, 1853) in the Cananéia estuary region, São Paulo, Brazil. Revista Brasileira de Biologia, 59(2), 183-194.

Hayes, A. J. S. (1999). Aspectos da atividade comportamental diurna da forma marinha do tucuxi "Sotalia fluviatilis" (Gervais, 1853) (Cetacea-Delphinidae), na Praia de Iracema (Fortaleza - Ceará - Brasil). Monografia de Licenciatura nãopublicada, Universidade do Algarve, Faro, Portugal.

Lodi, L. (2003). Tamanho e composição de grupos dos BotosCinza "Sotalia Guianensis" (Van Bénéden, 1864) (Cetacea, Delphinidae), na Baía de Paraty, RJ, Brasil. Atlântica, 25(2), 135-146.

Martinez, D. R., \& Klinchammer, E. (1969). A partial ethogram of the killer whale ("Orcinus orca"). Carnivore, 1, 13-27.

Miles, J. A., \& Herzing, D. L. (2003). Underwater analysis of the behavioral development of free-ranging Atlantic spotted dolphin (Stenella frontalis) calves (birth to 4 years of age). Aquatic Mammals, 29(3), 363-377.

Monteiro, M. S., Souto, A., \& Nascimento, L. F. (2006). Comparações entre os comportamentos de forrageio nas diferentes faixas etárias do boto-cinza (Sotalia guianensis) (Cetacea; Delphinidae) na Baía dos Golfinhos, Praia de Pipa - RN - Brasil. Revista de Etologia, 8, 13-25.

Muller, M. H., Boutiere, A., Weaver, A., \& Candelon, N. (1998). Ethogram of the bottlenose dolphin (Tursiops truncatus) with special reference to solitary and sociable dolphins. English Translation of Vie Milieu, 48(2), 89-104.

Nascimento, L. F. (2006). Boto cinza ("Sotalia guianensis" van Bénedén, 1864) (Cetacea, Delphinidae): Atividade aérea, forrageio e interações inter especificas, na Praia de Pipa (Tibau do Sul-RN) e um estudo comparativo entre duas populações do Nordeste do Brasil. Tese de Doutorado em Psicobiologia não-publicada, Universidade Federal do Rio Grande do Norte, Natal, RN.

Norris, K. S., \& Dohl. T. P. (1980). Behavior of the Hawaiian spinner dolphin, Stenella longirostris. Fisheries Bulletin, 77, 821-849.

Pizzorno, J. L. A., Siciliano, S., \& Simão, S. M. (1999) Quando os botos ganham uma identidade. Ciência Hoje, 25(150), 65-71.

Rossi-Santos, M. R. (1997). Estudo quali-quantitativo do comportamento de alimentação do golfinho ou boto cinza "Sotalia fluviatilis" Gervais, 1853 (Cetacea, Delphinidae) na Área de Proteção ambiental do Anhatomirim e Baía Norte de Santa Catarina. Monografia de Bacharelado em Ciências Biológicas não-publicada, Universidade Federal de Santa Catarina, Florianópolis, SC.

Shettleworth, S. J. (2001). Animal cognition and animal behaviour. Animal Behaviour, 61, 277-286.

Silva, V. M. F., \& Best, R. C. (1996). Sotalia fluviatilis. Mammalian Species, American Society of Mammalogists, 27, 1-17.

Simões-Lopes, P. C. (2005). O luar do Delfim: A maravilhosa aventura da História Natural. Joinville, SC: Letra D'água.

Slooten, E. (1994). Behavior of Hector's dolphin: Classifying behavior by sequence analysis. Journal of Mammalogy, 75(4), 956-964.

Spinelli, L. H. P., Nascimento, L. F., \& Yamamoto, M. E. (2002). Identificação e descrição da brincadeira em uma espécie pouco estudada, o boto cinza (Sotalia fluviatilis), em seu ambiente natural. Estudos de Psicologia, 7(1), 165-171. 
Nascimento, L. F., Medeiros, P. I. A. P., \& Yamamoto, M. E. (2008). Descrição do Comportamento de Superfície do Boto Cinza, Sotalia guianensis, na Praia de Pipa - RN.

Stevenson, M. F., \& Poole, T. B. (1976). An ethogram of the common marmoset, Callithrix jacchus, general behavioral repertoire. Animal Behaviour, 24, 428-451.

Tayler, C. K., \& Sayman, G. S. (1973). Imitative behavior by Indian ocean bottlenose dolphin (Tursiops truncatus) in captivity. Behavior, 44, 286-296.

Torr, G. A., \& Shine, R. (1994). An ethogram for the small scincid lizard Lampropholis guichenoti. Amphibia-Reptilia, 15, 21-34.

Würsig, B., Dorsey, E. M., Richardson, W. J., \& Wells, R. S. (1989). Feeding, aerial and play behaviour of the bowhead whales, Balaena mysticetus, summering in the Beaufort Sea. Aquatic Mammals, 15(1), 27-37.

Wedekin, L. L., Rossi-Santos, M. R., Bonin, C. A., Cremer, M., Lodi, L., Oliveira, F., et al. (2003, 26-29 junho). Análise comparativa do tamanho de grupo entre diferentes populações do boto-cinza, Sotalia guianensis (CETACEA: DELPHINIDAE), na costa do Brasil [Resumo]. In Resumos do II Congresso Brasileiro de Mastozoologia (pp. 57). Belo Horizonte, MG: Pontifícia Universidade Católica de Minas Gerais.

Yamamoto, M. E., \& Volpato, G. L. (2007). Comportamento animal. Natal, RN: Editora da Universidade Federal do Rio Grande do Norte. 